\title{
Apoyo a la Participación de Padres en las Escuelas Primarias: Un Estudio Etnográfico Sobre un Grupo Latinoamericano en Canadá
}

\author{
Judith K. Bernhard
}

Ryerson University

Marlinda Freire

University of Toronto

Veronica Pacini-Ketchabaw

University of Toronto

digital.library.ryerson.ca/object/370

Please Cite:

Bernhard, J. K., Freire, M., \& Pacini-Ketchabaw, V. (2000). Apoyo a la participación de padres en las escuelas primarias: Un estudio etnográfico de un grupo latinoamericano en Canadá. Education Policy Analysis Archives, 8, 52.

doi:10.14507/epaa.v8n52.2000

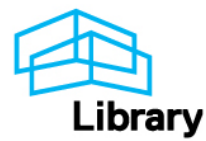




\title{
Education Policy Analysis Archives
}

Volume 8 Number 52

novembre 16, 2000

ISSN 1068-2341

\section{Apoyo a la participación de padres en las escuelas primarias: Un estudio etnográfico sobre un grupo latinoamericano en Canadá}

\author{
Judith K. Bernhard \\ Ryerson Polytechnic University
}

\author{
Marlinda Freire \\ Hospital for Sick Children, University of Toronto
}

Veronica Pacini-Ketchabaw

Ontario Institute for Studies in Education

University of Toronto

\begin{abstract}
Resumen
Este artículo describe cómo un grupo de padres latinoamericanos aprenden a manejar en forma más efectiva sus experiencias con el sistema educacional canadiense. La etnicidad y variables como sexo y clase social son determinantes críticos en las interacciones sociales, en las cuales los recién llegados constituyen el grupo minoritario. Durante ocho meses, doce padres latinoamericanos compartieron una vez al mes sus experiencias con la escuela. Finalizado el estudio se descubre que estos padres no sólo aprendieron a colaborar con los maestros sino que también los enfrentaron y validaron ante ellos sus diferencias etnoculturales. Esta interacción condujo a ganancias inesperadas más allá de lo relativo al proceso educacional. A través de este examen los padres develaron lo que el sistema escolar considera como intervención familiar ideal, sin menoscabo del bagaje cultural del estudiante. Este estudio puede ser un modelo de adaptación para grupos de recién llegados que intentan integrarse al sistema educativo.
\end{abstract}

\begin{abstract}
This article describes how a group of Latin American parents became more effective in their dealings with their children's schools, a mainstream Canadian institution. Ethnicity, along with race, gender, and social class, is a critical determinant in of the interactions between schools and any group of newcomers to a society, particularly when those newcomers are an ethnic minority. Over an eight-month period,
\end{abstract}


twelve Latin American parents met monthly to discuss aspects of their children's experience with the Canadian educational system. These parents learned to collaborate with teachers and expressed their needs, but also affirmed their ethno-cultural differences. The positive feedback on their activities led to unforeseen gains, not just in relation to education and the schools. This exploratory study focuses on how the experience helped the parents to better comprehend what is expected of them in the support of their children's schooling while retaining their own cultural assets. This study may serve as a possible model of adaptation for newcomer groups in their efforts to integrate in the school system.

\section{Introducción}

Gobiernos, ministerios y escuelas en Canadá, como en otros países del mundo, enfatizan la importancia de la colaboración entre la familia y la escuela. Nuestro trabajo con un grupo de padres latinoamericanos residentes en Canadá, efectuado entre octubre de 1997 y junio de 1998 demuestra que, desafortunadamente, en este país existen dificultades para la implementación de la legislación pertinente. En este artículo, basado en un estudio etnográfico de un grupo latinoamericano en Toronto, exploramos las experiencias de las familias latinoamericanas y las dinámicas observadas dentro del contexto educacional canadiense. Documentamos además, desde su propia perspectiva, la naturaleza de las desventajas institucionales a las que estos padres se enfrentan. Asimismo, discutimos cómo el proceso de grupo facilitó la participación eficaz de los padres en las experiencias educacionales de sus hijos. Examinamos las dificultades de los padres en su adaptación al sistema educacional como un aspecto más de las dificultades globales que los inmigrantes experimentan en sus intentos de integración a la sociedad mayoritaria. (Freire, 1993). Esta investigación y la información obtenida en este trabajo permitieron encarar las inquietudes siguientes y demostrar cómo los padres, trabajando colectivamente, resolvieron estas inquietudes de una manera más efectiva:

1. ¿Cómo las familias latinoamericanas perciben la práctica escolar?

2. ¿¿De qué manera los padres latinoamericanos perciben el sistema escolar y cuáles son los roles sociales que les han sido asignados?

3. ¿De qué manera los padres latinoamericanos enfrentan un proceso institucional que los pone en desventaja por ser emigrantes y tener un manejo limitado del idioma dominante del país que los recibe?

Además de intentar aclarar la dinámica de interacción minoría-mayoría dentro de un encuadre escolar, este trabajo describe el proceso de grupo y la transformación que ulteriormente permite a los padres enfrentar, más preparados, el futuro académico de sus hijos, y a ellos ejercer más eficazmente su potencial de poder sociopolítico y económico en la nueva sociedad, basándose en sus interacciones con el sistema escolar.

De acuerdo con la última estadística disponible, la proporción de población que 
habla español como primera lengua en Canadá ha aumentado en más del doble en la última década. De 70,000 en 1981 a 187,000 en 1996 (Statistics Canada, 1998). De acuerdo con un estudio reciente, los inmigrantes latinoamericanos integran uno de los dos grupos étnicos con más probabilidades de vivir en la pobreza en Canadá (Halli \& Kazemipur, 1997). Aunque el latinoamericano es uno de los grupos con mayor crecimiento en este país, al estar diseminado a través de numerosas escuelas, pareciera representar una pequeña minoría.

\section{Marco teórico}

Nuestro marco teórico es social y ante todo estructural. Las desventajas sistémicas son consideradas múltiples, basadas en factores como clase social, raza, género y tipo de inmigración (Apple, 1992; Ng 1993). El poder es visto como esparcido en instituciones oficiales y en las acciones cotidianas de la sociedad. (Bourdieu, 1986; Cannella, 1997; Looker, 1994). Ng (1987) propone que clase social, raza, género y condiciones de inmigración están constituidas por relaciones y prácticas sociales institucionalizadas. En particular, el origen étnico no está considerado como una característica inherente al grupo, sino ante todo, como una continua imputación e interpretación por aquellos que se constituyen a sí mismos como grupo dominante. Estos mismos argumentos se aplican para la categoría de "razas" (Dei, 1993a, 1993b; Miles,1989).

Usamos el concepto de "capital cultural" de Bourdieu (1986) al referirnos al modo de ser, conocimientos, habilidades, disposiciones y capacidades que establecen a una persona en un contexto y estrato social determinados dentro de un ambiente de relaciones sociales. A través de esas relaciones, la misma persona es vista de acuerdo con su posición social previamente estructurada. En nuestro trabajo anterior con familias latinoamericanas encontramos que frecuentemente hay una disparidad de valoración entre el capital cultural que las familias traen a la nueva situación (país, sociedad, escuela, etc.) y aquel (implícitamente) requerido por la escuela. La valoración de los profesores sobre el "apoyo de los padres" al desarrollo académico de sus hijos resultó estar altamente determinada por modelos específicos de colaboración padre- hijo en la cultura mayoritaria. Sin embargo, el quehacer de los padres, incluyendo los consejos y la orientación que le dan a los niños, está basado en su propia visión cultural y en lo que a ellos les corresponde hacer en dichas situaciones.

Aunque podríamos llamar a esto una simple situación de desequilibrio en las habilidades y la manera de ver un mismo proceso por dos agentes diferentes, tal "desequilibrio" representa, en nuestra opinión, un fenómeno mucho más complejo, ya que está relacionado con diferencias de poder a nivel sociopolítico y económico.

Lareau (1989), en su trabajo con comunidades en Estados Unidos, encontró que los padres de clase social alta sabían utilizar el sistema y conseguían que los maestros ajustaran el programa escolar de acuerdo con las necesidades de sus niños. En cambio, las familias de clase socio económica baja no logran tales ajustes a pesar de que las necesidades de estos niños son mayores. En vista del bajo rendimiento escolar de los niños latinoamericanos en Canadá (Bernhard \& Freire, 1996; Brown, 1994, Drever, 1996), consideramos que es importante que los padres entiendan cómo funciona el sistema educacional canadiense y la habilidades que se requieren para ser más eficaces en sus interacciones con el mismo. 
El segundo marco de referencia en este trabajo es la teoría ecológico-cultural. Spindler (1990) ha considerado la escolaridad como un "proceso cultural obligatorio". De acuerdo con esta posición teórica, los profesores, reflejando una posición etnocéntrica, transmiten los valores dominantes e inadvertidamente debilitan la identidad cultural de los estudiantes de grupos minoritarios. Al sentir su propia identidad en peligro, los estudiantes responden con conductas defensivas que contribuyen a perpetuar su marginación (Trueba, 1993). Las teorías de deficiencia cultural invierten la relación entre causa y efecto (Barrera, 1997) y por lo tanto refuerzan posiciones de victimización de grupos minoritarios.

Nuestro tercer marco teórico es la teoría anti-racista (e.g., Dei, 1993a). Este marco referencial es fundamental en el análisis de nuestro trabajo. Es imposible cambiar el rendimiento académico de los niños sin antes entender el contexto en el cual ellos viven sus experiencias, incluyendo las determinadas por el color de la piel. La primera serie de variables contextuales que identifican subordinación son raza, clase social y género. El problema de la interacción entre raza y clase social es sumamente complejo y ha sido explorado por numerosos autores (Bernhard, Freire \& Pacini-Ketchabaw, in press; Dei, 1993a; Ng, 1993). En el contexto del presente estudio, vemos la subordinación como la continuidad de un proceso histórico, complejo e impersonal con implicaciones mayores de factores como raza, etnicidad, clase social y género.

Algunas de las dificultades que los padres encuentran se deben, en parte, a la exclusión social por parte del grupo dominante y al hecho de que no existe una red informal de comunicación a través de la cual los padres puedan compartir información importante y adecuada sobre la práctica escolar en el nuevo país. Los padres asumen que el sistema educacional canadiense funciona en forma similar al de su país de origen.

Más aún, los padres se sienten intimidados por las autoridades escolares basados en sus experiencias previas con instituciones oficiales en su país de origen. Estas dificultades son agravadas por las barreras idiomáticas.

Es posible, entonces, tratar de identificar, analizar y ayudar a vencer las dificultades escolares de los niños, formando grupos de padres con un bagaje cultural y lingüístico similar y a través de facilitadores de grupo que funcionen como mediadores culturales. Es fundamental que estos mediadores compartan la cultura y el idioma nativo de las familias, entiendan cómo funciona el nuevo sistema escolar y conozcan las estrategias que permiten una colaboración efectiva de los padres en su tarea de apoyar a los niños en la escuela. Es de primordial importancia que los padres comprendan que adquieren una gran ventaja al participar activamente en el quehacer escolar. Posiblemente esto último fue una meta mayor implícita en la elaboración de este proyecto.

\section{Método}

Durante un período de ocho meses, un grupo de doce padres latinoamericanos se reunieron una vez por mes. Aunque hubieron padres que asistieron a algunas de las reuniones, fueron las madres las que participaron regularmente y es por eso que en el resto de este artículo usamos solamente el término "madre". El grupo estaba compuesto por cuatro madres chilenas, una argentina, tres salvadoreñas, dos uruguayas, una mexicana y una nicaragüense. Nueve de las madres eran de clase trabajadora y 
solamente tres de clase media. Las edades fluctuaban entre los 33 y los 42 años. Todas tenían algún nivel de educación secundaria y tres de ellas, educación universitaria. Todas estas madres constituían familias intactas (madre y padre). En este grupo, las madres sudamericanas tenían un promedio de 15 años de residencia en Canadá, mientras que las centroamericanas eran de inmigración más reciente, con un promedio de 8 años, y una de ellas había residido menos de un año en el país. Todas tenían, por lo menos, un niño en edad escolar primaria. Las edades de ellos fluctuaban entre los 18 meses y los 16 años. El trabajo se enfocó en la experiencia escolar de los niños entre los 4 y 14 años, con el supuesto adicional de que cualquier aprendizaje de las madres sobre el sistema escolar las ayudaría con los niños menores de 4 y mayores de 14 años. Estos niños asistían a escuelas con un perfil étnico muy diverso y donde la población latinoamericana no era, en la mayoría de los casos, más del 2 o 3 por ciento.

Solamente dos de las madres tenían hijos en una misma escuela en que la población latina constituía el 15 por ciento del estudiantado. En esta escuela en particular había un profesor latinoamericano encargado del programa de "Herencia Lingüística" (español). Las madres que asistieron fueron contactadas a través de asociaciones comunitarias e invitadas a participar en esta serie de reuniones de grupo. Se les informó que el proyecto constituía un trabajo de investigación y cuáles serían los posibles beneficios para los padres participantes y para la comunidad latinoamericana en general. Las reuniones fueron de dos horas de duración, conducidas en español y se ofreció cuidado de niños y refrigerios.

Los dos investigadores principales asistieron a todas las reuniones. La facilitadora, entre otras funciones, iniciaba cada sesión, hacía un resumen de los temas discutidos en las sesiones previas, invitaba a los participantes a comentar sobre este resumen y finalmente, iniciaba la discusión de la nueva sesión con una pregunta neutra sobre un tema abierto pero pertinente. La sesión podía dedicarse a temas previamente discutidos que necesitaban más elaboración o podían ser temáticas nuevas que reflejaban otras áreas de preocupación de las madres con respecto a la educación de sus niños y el sistema educacional. De este modo las participantes fueron capaces de reflexionar de manera continua durante el periodo que duró el proyecto, sobre sus contribuciones y cuestionamientos y al mismo tiempo, de incorporar nuevos elementos que les permitían, no solamente entender el sistema escolar, sino reafirmarse frente al mismo. En forma rotativa, un co-facilitador, elegido entre las madres del grupo, estaba encargado de dirigir la discusión. Inicialmente las madres se resistían a participar como cofacilitadoras pero pronto se notó un cambio de actitud con un aumento en la confianza para ejercitar dicho rol. Cada sesión fue grabada, transcrita y codificada.

El análisis preliminar de este trabajo fue presentado al grupo para su verificación. En la segunda etapa, los temas identificados como centrales en el trabajo con los padres fueron discutidos informalmente con dos expertos en el área para corroborar la validez y la relevancia de los mismos. La transcripción de las grabaciones permitió hacer un informe sobre las historias y versiones de las madres en la descripción de las experiencias de sus hijos y de las propias con los maestros y autoridades del sistema escolar. Estas madres constituyen una muestra de conveniencia, por lo tanto cualquier intento de generalizar nuestras conclusiones debería hacerse (y lo hacemos) con toda cautela. El propósito del trabajo fue documentar cómo este grupo particular de madres latinoamericanas consiguió entender mejor el sistema escolar canadiense, lo que los 
profesores y la escuela valoran y esperan del niño y de la familia, expresar con más confianza las necesidades de sus niños, y la forma más efectiva de intervenir a nivel escolar en favor de ellos.

\section{Resultados}

\section{A. Descripción del grupo y las experiencias de las madres con el sistema educacional}

Al comienzo del estudio las madres estaban aisladas y cada una vivía sus experiencias en forma individual. El grupo sirvió como medio para conectarlas a través de los problemas comunes en su contacto con el nuevo sistema educacional. Estas madres aprendieron a comunicarse más efectivamente con los maestros y descubrieron las expectativas tácitas que los maestros esperan encontrar en los "padres eficaces". En el proceso de reunirse para discutir la educación de sus hijos, estas madres comenzaron a reconocer los aspectos de la situación educacional de sus niños que reflejaban el funcionamiento del sistema. Alcanzando más allá de sus experiencias individuales, comenzaron a apoyarse mutuamente. A decir lo que pensaban, (inicialmente en el grupo y más tarde en las escuelas) y a exigir que algunos de los programas educacionales se adaptaran a las necesidades de sus hijos. Por ejemplo, la señora Morales acota: "le dije al maestro que quería que mi hijo fuera al programa de francés, respondió que no porque no sabía suficiente inglés. Pero le dije al maestro, ¿cuál es la diferencia si inglés tampoco es su primera lengua?. Entonces el maestro arregló una reunión especial con el director, un representante del Consejo de Educación, una sicóloga y una trabajadora social. Cuando llegué a la reunión tenía miedo pero de todas formas les dije lo que quería y ellos lo aceptaron". Las madres en forma paralela experimentaron un proceso de organización de un grupo de autoayuda que reforzó su autoestima y les ayudó a vislumbrar la posibilidad real de comenzar a entender el sistema escolar con su agenda oficial y las estructuras que permiten mantener el status quo de las clases privilegiadas.

Como grupo empezaron a entender la situación de riesgo implícita en su posición de nuevos inmigrantes o refugiados de países en desarrollo. Las madres comenzaron a experimentar un proceso de aprendizaje que afirmó la base para un proceso de concientización como el descrito por Paulo Freire (1972). Además, se sintieron capaces de iniciar cambios y se fueron dando cuenta de su potencial y de su efectividad ante situaciones que antes les parecían impenetrables. A partir de entonces, empezaron a difundir este conocimiento con otras madres latinoamericanas que no asistían al grupo pero que compartían "desconocimientos" y preocupaciones parecidas. Por ejemplo, la señora Díaz dijo: "Mi comadre no puede asistir a estas reuniones pero yo le hago saber todo lo que aquí discutimos. Entre las dos tratamos de entender más aún. Ella quiere pedirle a su hermana que también se junte con nosotras porque sus hijos son chiquitos pero ya tienen problemas en la escuela. Yo no sé cómo podemos tratar de defendernos mejor de las cosas que les pasan a nuestros niños en la escuela sin saber lo que estamos aprendiendo en este grupo".

Durante la participación de las madres en el grupo vimos varios casos de madres que cuando entraban a las escuelas se sentían incómodas, ignoradas y consideraban que asistir a la escuela no era efectivo para las necesidades de ellas y de sus hijos. Por 
ejemplo, la señora Blanco dijo al comienzo del proyecto: "Cuando voy a las reuniones sobre calificaciones, salgo con muchas dudas, me voy de la reunión tal como llegué, sin entender nada. Los profesores asumen que entendí todo. Uno siente que en lo que dicen los maestros no hay nada que discutir, especialmente si ellos consideran que tu hijo no tiene problemas serios. Ellos te entregan las notas, asumen que tu entendiste y no dan oportunidad de preguntar nada. Entonces me voy como si hubiera entendido lo que dijeron pero en realidad no entendí nada". La señora Castillo describió su experiencia con el siguiente comentario: "En la escuela de mi hijo, los padres no van a la sala de clases. La maestra recibe a los niños en la mañana y los lleva a la sala de clases. Yo no tengo oportunidad de hablar con ella. Me gustaría saber por qué mi niño está en una clase donde ella enseña dos cursos, pero no sé si preguntarlo. No me atrevo a decir: quiero que lo pongan en una clase regular solamente con niños del mismo curso. La maestra sabe por qué lo puso en esa clase. No quiero tener problemas con ella, pero me preocupa bastante". A medida que el grupo progresaba, estas mismas madres comenzaron a interactuar con los maestros, a reafirmarse a sí mismas y a saber que sus opiniones tenían valor y podían ser escuchadas. La señora Rojas dijo: "Esta es la primera vez que participo en un grupo con esta dinámica. Tenemos la oportunidad de tratar los temas en profundidad. Como no somos muchos tenemos la oportunidad de hablar, lo que me ha ayudado a sentirme parte del grupo. Hemos compartido temas que son nuestros".

En el proceso de reunirse, las madres empezaron a valorarse a sí mismas y lo que ellas, u otros, pudieron haber considerado deficiencias, comenzaron a desaparecer y a transformarse en fortalezas y capital instrumental. Seis semanas más tarde, en algún momento del proceso, la señora Blanco dijo: "Este grupo me ha servido para ayudar a mis amigos que tienen problemas con sus niños en la escuela. Yo les digo: mira, tú puedes venir aquí, o puedes hacer esto o aquello. Les da fuerza. Esto me ha ayudado a compartir con otros padres ya que hay tanta gente que no sabe como enfrentar este tipo de situaciones". La señora López orgullosamente anunció en una de las reuniones: "He decidido que voy a empezar a ir a la escuela y molestar a los profesores hasta que me escuchen. Estoy en eso y si todos los padres hacemos lo mismo terminarán escuchándonos. El otro día la profesora me pidió ir a la escuela para ayudar a los niños con la lectura". también fue interesante observar que las madres describieron cómo el proceso que se daba en el grupo les ayudó a generalizar en otras tareas sobre sus propios derechos y los de sus hijos. La señora González dijo: "El grupo me ayudó a entender a mi hija. Me ayudó a ver que tenía ciertos derechos y que eran legítimos. Me ayudó a entender que la persona que estaba enseñando a mi hija no era un ser superior que yo no podía alcanzar ni hablarle. El maestro y yo tenemos la misma tarea, la educación de mi hija".

\section{B. Subordinación y reconocimiento de las desventajas}

Otros factores que las madres presentaron como problemáticos fueron las diferencias que percibían en las áreas de género y raza. En cuanto a las diferencias de género las madres habían comprobado que la mejor estrategia para ser escuchadas por las autoridades escolares era ir acompañadas por el esposo u otro varón allegado a la familia. Algunas madres describieron cómo, a pesar de ser ellas las que verbalizaban el 
problema, el personal escolar normalmente se dirigía y hacía contacto visual con el varón. La señora Godoy dijo: "Han habido circunstancias en que he ido a la escuela con mi esposo para hablar con los profesores y he notado una mejor disposición de parte de ellos porque él estaba presente. Una vez le preguntaron su opinión y le dieron opciones, lo que no pasa si voy sola. Cuando voy a las reuniones con mi esposo los profesores tienden a dirigirse solo a él y no a los dos".

En cuanto a raza, en la mayoría de los casos, las participantes no elaboraron sus dificultades en términos de "problemas raciales". Las madres no se veían a sí mismas correspondiendo a un encuadre racial negro/caucásico u otro. Las madres pertenecían a diferentes grupos raciales y poseían distintos tonos de piel. Sin embargo, muchas de ellas se referían continuamente a la población latinoamericana como una raza y no como un grupo étnico. Algunas madres de piel más oscura mencionaron como factor adicional en las dificultades que los niños o ellas habían tenido, el racismo que habían experimentado en las instituciones escolares y que ellas atribuían, particularmente, al color de su piel. La señora Godoy dijo: "Pienso que nuestra posición en la sociedad es clara, tenemos desventajas debido al color de nuestra piel y a nuestro lenguaje y que nuestros niños tienen las mismas desventajas en la escuela. Un día con mi hija nos encontramos con dos nativos canadienses y ella les preguntó si eran peruanos ya que se veían como nosotros.... Muchas veces nos sucede que la gente piensa que somos nativos canadienses cuando en realidad somos latinos. Aquí sabemos lo que maltratan a los nativos canadienses".

La raza, de la manera presentada por las madres, es considerada en este trabajo como una construcción social, más que biológica o antropológica. Para estas familias, la cuestión del color de la piel no es vista como un indicador primario de la raza. De la misma manera, el ancestro latinoamericano y el bagaje de experiencias son identificados por el grupo como raza. Aunque en los Estados Unidos el discurso político reconoce los distintos grupos étnicos, particularmente negros y blancos, nuestros resultados demuestran que en Canadá la situación de los latinoamericanos pareciera ser conceptualizada en forma diferente. Las madres no se consideraban a sí mismas como pertenecientes a un determinado grupo racial, pero a medida que el trabajo de grupo progresó, ellas comenzaron a ver sus semejanzas y sus ancestros comunes, y lo empezaron a definir como "raza".

Los latinoamericanos en Canadá recién comienzan a tomar conciencia de grupo étnico. Basados en el informe de las madres, no existen evidencias de que las escuelas canadienses hayan reconocido a las familias latinoamericanas como un grupo étnico particular, pero las madres describen experiencias discriminatorias en relación con diferentes aspectos del quehacer escolar que relacionan con raza. Por ejemplo la señora Pérez dice: "a veces la discriminación es muy sutil, especialmente con los niños que están empezando a hablar inglés y los ponen en niveles básicos aún cuando ellos son capaces para un nivel más avanzado. Como padres, nosotros sabemos que nuestros hijos son capaces pero los maestros insisten en que deben ir a los niveles básicos. A veces, solamente porque nos vemos y hablamos diferente. Entonces nosotros tenemos que luchar por sus derechos". La señora Mendoza agregó: "La escuela de mi hijo tiene principalmente una población portuguesa, italiana e hispana, sin embargo la mayoría de los maestros no tienen ancestro latino, entonces, se puede ver favoritismo con los niños de piel mas clara". Esto va en contraste con la situación que se vive en los Estados 
Unidos donde la población de color, incluyendo los latinoamericanos, está claramente definida como un grupo étnico reconocido. Como resultado, ciertos grupos étnicos sufren una invalidación del sentido de sí mismos que los puede llevar a un racismo internalizado como un factor adicional en la perpetuación de un desequilibrio en el poder socio-político y económico ya establecido por el sistema dominante.

Cuando el grupo dominante determina cuál es el capital cultural predominante y por lo tanto, el que se valora preferencialmente, las diferencias culturales se vuelven "deficiencias" de acuerdo con las medidas estándar de lo que se considera "normal y valioso" en ese medio. Los profesores, inadvertidamente, están incapacitados para evaluar el conocimiento y el capital cultural que las familias de clase trabajadora y de grupos minoritarios poseen en el momento de tratar de insertarse en el medio dominante. Dentro de este contexto y de acuerdo con el criterio institucional, no se reconoce la voluntad de colaboración y los esfuerzos de las familias en apoyar el desarrollo académico de los niños en la forma que ellos culturalmente lo entienden.

Los profesores, sin suficientes recursos personales ni instrumentales para tener contacto individualizado con sus estudiantes o con las familias, corren el peligro de convertirse en simples agentes transmisores del punto de vista etnocéntrico dominante. En estas circunstancias, las familias se enfrentan con obstáculos estructurales prácticamente insalvables que no les permiten remover las barreras discriminatorias para lograr una verdadera colaboración y un trabajo en conjunto con las escuelas. Las limitaciones lingüísticas del uso del segundo idioma, el desconocimiento de los programas escolares y de la operación y funcionamiento del nuevo sistema escolar, son tres aspectos básicos que tienden a magnificar y perpetuar los obstáculos estructurales.

A través del proceso de crecimiento que las madres experimentaron, las ganancias parecieron mayores que las esperadas inicialmente para el proyecto. Por ejemplo la señora Rojas dijo: "Estaba preocupada por el rendimiento de mi hijo en matemáticas. Sé que necesita apoyo, entonces fui a la escuela y la profesora me dijo que no necesitaba clases especiales porque alguien en su curso lo estaba ayudando. después de eso me dejé de preocupar, pero ahora que terminó la escuela me mandaron un libro entero para que trabaje en el verano ya que estaba atrasado en matemáticas. Ella debería haberle dado tareas diarias y no esperar hasta el final. No me parece correcto. Si hubiera sabido habría insistido pero la profesora me dijo que no me preocupara. La próxima vez no me voy a quedar tan tranquila, voy a insistir hasta que yo me convenza que mi hijo lo está haciendo bien". De la señora Castillo escuchamos lo siguiente: "Nos juntamos con Esther y fuimos a una charla política en la universidad... Era la primera vez que participábamos en algo así. No fue fácil pero la participación en este grupo nos dio confianza para enfrentar nuevos niveles de actividad". La señora Morales acotó: "Una vez la secretaria de la escuela me llamó para que vaya a buscar a mi hijo porque se había pintado la cara y estaba distrayendo a los otros niños de la clase. Dijo que mi hijo estaba fuera de control y que lo tenía que ir a buscar inmediatamente. Me dijo que ellos no sabían que otra cosa hacer. Cuando la escuché sentí como si alguien me hubiera vaciado un balde de agua fría en la cabeza. Me sentí choqueada y avergonzada. No pude contestar nada y colgué. En otro momento habría salido corriendo a hacer lo que la secretaria me pedía. Esta vez fue distinto, me senté en la cama, estaba temblando, respiré profundo, llamé a la secretaria de vuelta y le dije no iría a recoger a mi hijo, que no pensaba que lo que el niño estaba haciendo fuera tan terrible". 


\section{Discusión general}

Durante nuestras primeras reuniones, las madres estaban frustradas por el bajo rendimiento de sus hijos y porque además sentían que sus inquietudes y cuestionamientos no eran bien recibidos. Ellas, hasta el momento de empezar a participar en el grupo, habían reaccionado pasivamente como respuesta a su percepción de los maestros como figuras de expertos, autoritarias e inaccesibles. Por su desconocimiento del modus operandis del nuevo sistema escolar y por la falta de éxito en las iniciativas que ellas habían tomado frente a las problemáticas presentadas.

Como resultado del trabajo de grupo, las madres comenzaron a tomar iniciativas efectivas y a hacerse oír a medida que cumplían exitosamente con varios objetivos relacionados con las escuelas. Esta retroalimentación positiva fue fundamental, ya que la mayoría manifestaron una necesidad de participar en forma más activa en las organizaciones latinoamericanas con representación oficial frente al sistema escolar, lo cual podría, a más largo plazo, ayudar a operar los cambios estructurales que sean necesarios. Consiguieron entender mejor el rol de los consejos escolares y se sintieron más preparadas para participar en forma activa y eficaz.

Los ejemplos presentados en este artículo demuestran la complejidad subyacente de las interacciones entre las familias latinoamericanas y las escuelas canadienses. Por ejemplo, si por una parte, existía una "falta de participación de los padres" de acuerdo al personal de la escuela, esto no debería ser entendido, automáticamente, como una falta de interés o motivación por parte de ellos en el desarrollo académico de sus niños. Más bien deberíamos entender estas acciones como el resultado de la percepción del nuevo sistema escolar por parte de los padres y de la interpretación que los maestros dan a su rol en la educación de todos sus estudiantes, incluyendo los hijos de emigrantes. Es nuestra opinión que éste, como parte de un proceso global de integración de los recién llegados, es un proceso bidireccional en el cual la mayor responsabilidad le corresponde al sistema ya establecido (sistema escolar).

Si bien el presente proyecto fue dirigido al nivel de educación primaria, la información obtenida indica que, según la perspectiva de los padres, ciertos modelos de comportamiento entre los padres y el sistema escolar han sido establecidos antes que el niño comience su educación primaria. La marginación del capital cultural de los padres es, realmente, un fenómeno que tiene sus orígenes en interacciones anteriores al comienzo de la educación escolar formal. En un estudio con pre- escolares latinoamericanos que asistían a guarderías asociadas a programas de aprendizaje de inglés para sus padres, se encontró, de parte de las educadoras, una devaluación total del uso del idioma español y de las habilidades de estos padres para criar a sus hijos (Benhart y Freire, 1996). Cualquier propuesta genuina de cambio tendría que comenzar al nivel de las guarderías infantiles, ya que éste es el primer contacto de los padres con el sistema educativo (Lee \& Seiderman, 1998). Si esto se hace en las primeras etapas del desarrollo del niño y de su contacto con los sistemas oficiales, los grupos de padres podrían desarrollar un sistema de apoyo y conocimiento que les permita mantenerse unidos y mejor capacitados para aportar más efectivamente en la formación académica de sus hijos.

Los padres pueden sólo hacer el trabajo que a ellos corresponde. El aspecto de 
comprensión y reconocimiento de otros grupos étnicos y la práctica de los educadores necesita ser dirigido hacia otras alternativas de trabajo cuyas responsabilidades le corresponden al sistema educacional.

¿Cómo se explica la falta de interacción bidireccional en la cual padres y maestros se reúnan y escuchen las preocupaciones de unos y otros? El sistema educacional asume un modelo clásico de interacción: Los maestros convocan a reuniones y conferencias entre padres y maestros, con una agenda unidireccional. Este proceso transforma a los padres en agentes impotentes (Dehli, 1994; MacLure \& Walker, 1998). Generalmente las escuelas esperan que los padres acudan a ellas y reciban lo que se les ofrece, en un formato técnico, generalmente incomprensible para los padres.

Alternativamente, se espera que los padres hagan una presentación elaborada, respaldada por una evaluación profesional de los servicios adicionales requeridos por sus hijos en la escuela. Esto solamente es posible para los padres de clase media/alta familiarizados con el sistema y que cuentan con los medios económicos que les permiten documentar las dificultades que sus niños experimentan, en forma privada si ello es necesario.

Los padres no podrán efectuar cambios significativos hasta que las estructuras se conviertan en una interacción de dos vías donde ambos lados puedan hablar y escucharse, y donde los padres tengan alguna certeza de que sus preocupaciones y las sugerencias de cómo enfrentar las dificultades serán implementadas o por lo menos exploradas. Esta situación anómala se debe a obstáculos institucionales, más bien que a acciones individuales mal intencionadas.

La escasez o ineficacia de mecanismos y/o recursos, para involucrar a los padres e invitar sus aportes, van probablemente a derivar en la clase de dificultades que hemos detallado, en una población compuesta por diversos grupos étnicos. Un componente clave para establecer un modelo de colaboración, más allá de lo que los padres mismos pudieran hacer, requiere que los maestros desarrollen una mayor comprensión de la cultura y de las preocupaciones de las familias y que obtengan experiencia práctica trabajando directamente con los padres (Corson, Bernhard \& González-Mena, In press; Moll, Amanti, Neff \& González, 1992). también es fundamental que los educadores entiendan el valor del idioma nativo, no solamente como puente en la adquisición de un segundo idioma, sino como la base del desarrollo global del niño (Freire M., Benhard J., 1997).

En nuestra opinión, los maestros y la escuela pueden jugar un rol de importancia fundamental en crear o facilitar nuevas disposiciones conducentes a una experiencia escolar positiva para las familias inmigrantes y sus niños en el intento de integrarse a la nueva sociedad. Como resultado de este trabajo, presentamos aquí una experiencia y una forma alternativa de participación de los padres dentro del nuevo sistema escolar. Este tipo de participación reconoce el contexto histórico-cultural particular en el cual las familias viven su realidad actual y se basa en la comprensión básica de los padres del proceso institucional dominante, en un área fundamental e ineludible: la educación. En resumen, este proyecto trata de facilitar un proceso a través del cual los padres se transformen en agentes activos y conscientes de una pedagogía liberadora que pudiera llevar a transformaciones substanciales, no solamente en estos padres y sus hijos, sino también en las generaciones futuras. 


\section{Referencias}

Apple, M. (1992). The text and cultural politics. Educational Researcher, 21(7), 4-11.

Barrera, M. (1997). A theory of racial inequality. In A. Darder, R. Torres and H. Gutiérrez (Eds.), Latinos and education: A critical reader (pp. 3-44). New York: Routledge.

Bernhard, J.K., Freire, M. \& Pacini-Ketchabaw, V. (In press). A Latin American parentsí group participates in their childrenís schooling: Parent involvement reconsidered. Canadian Ethnic Studies Journal.

Bernhard, J.K., \& Freire, M. (1996). Latino refugee children in childcare: A study of parents and caregivers. Canadian Journal of Research in Early Childhood Education, 5(1), 59-71.

Bourdieu, P. (1986). The forms of capital. In J. C. Richardson (Ed.), Handbook of theory and research in the sociology of education (pp. 241-257). New York: Greenwood Press.

Brown, R. S. (1994). A follow-up the Grade 9 Cohort of 1987 secondary students survey participants. Toronto, Canada: Toronto Board of Education.

Cannella, G. S. (1997). Deconstructing early childhood education: Social justice and revolution. New York: Lang.

Corson, D. (1998). Changing education for diversity. Philadelphia, PA: Open University Press.

Corson, P., Bernhard, J. K., \& Gonzalez-Mena, J. (In press). Culturally situated explorations of child development: A home visit project for prospective early childhood teachers. Journal of Early ChildhoodTeacher Education.

Dehli, K. (1994). Parent activism and school reform in Toronto. Unpulblished Doc. Department of Sociology in Education, OISE.

Dehli, K. (1987). 'Ethnicí 'parentí 'communityí: The proper channeling of education politics. In J. Young (Ed.), Breaking the mosaic: Ethnic identities in Canadian schooling (pp. 224-239). Toronto, ON: Garamond Press.

Dei, G. (1993a). The challenges of anti-racist education in Canada. Canadian Ethnic Studies, 25 (2), 36-51.

Dei, G. (1993b). Narrative discourses of black/African- Canadian parents and the Canadian public school system. Canadian Ethnic Studies, 25 (3), 45-65. 
Drever, N. (1996). Frustrated learners: An ethnographic study of Spanish-speaking students at an inner-city high school in Toronto. Unpublished master's thesis, Toronto, ON: University of Toronto.

Freire, M. (1993). Mental health, culture, children and youth. In R. Masi, L. Mensah, and K.A. McLeod (Eds.), Health and cultures: Exploring the relationships, Vol. II,Toronto.

Freire, M.; Benhard, J.K. (1997). Caring for and Teaching Children Who Speak Other Languages. Include Me Too. Human Diversity in Early Childhood.(Chapter 9, pp 160).

Freire, P. (1972). Pedagogy of the oppressed. New York, NY: Continium.

Kazemipur, A. Halli, S. S. (1997). Plight of immigrants: The spatial concentration of poverty in Canada. Canadian Journal of Regional Science, 20 (1,2), 11-28.

Lareau, A. (1989). Home advantage: Social class and parental intervention in elementary education. New York: The Falmer Press.

Lee, L., \& Seiderman, S. (1998). Families matter: The Parent Services Project. Boston, MA: Harvard Family Research Project.

Looker, D. E. (1994). Active capital: The impact of parents on youthsí educational performance and plans. In L. Erwin \& D> MacLennan (Eds.), Sociology of education in Canada (pp. 164-187). Toronto, ON: Copp Clark Longman.

MacLure, M. \& Walker, B. M. (1998). Disenchanted evenings: The social organization of talk in parent-teacher consultations in UK secondary schools. Paper presented at the American Educational Research Association meeting, San Diego: April.

Miles, R. (1989). Racism. London: Routledge.

Moll, L. C., Amanti, C., Neff, D., \& Gonzalez, N. (1992). Funds of knowledge for teaching. Using a qualitative approach to connect homes and classrooms. Theory into Practice, 31, 132-141.

Ng, R. (1987). Ethnicity, schooling and the social division of labour: A response to Jackson. In J. Young (Ed.), Breaking the mosaic: Ethnic identities in Canadian schooling, (pp. 183-189). Toronto: Garamond Press.

Ng, R. (1993). Racism, sexism, and nation building in Canada. In C. McCarthy and W. Crichlow (Eds.), Race, identity and representation in education (pp. 50-59). New York: Routhledge.

Spindler, G., \& Spindler, L. (1990). The American cultural dialogue and its transmission. London: Falmer.ddd 
Statistics Canada (1998). Daily. Ottawa: Supply/Services Canada.

Trueba, H. (1993). Cultural diversity and conflict: The role of educational anthropology in healing multicultural America. In P. Phelan \& A. L. Davidson (Eds.), Renegotiating cultural diversity in American schools. New York: Teachers College Press.

\section{Acerca de los Autores}

Judith K. Bernhard

Ryerson Polytechnic University

bernhard@ryerson.ca

Dr. Judith Bernhard is a native of Chile, a lecturer, author, educator, and parent. She is currently an associate professor at the School of Early Childhood Education at Ryerson University, Canada. Over the last 20 years, she has been involved in a number of initiatives including a national study on diversity (Paths to Equity: Cultural, linguistic, and racial diversity in Canadian Early Childhood Education) and the establishment of the Early Childhood Diversity Network. Dr. Bernhard teaches child development courses and has a particular interest on infants, toddlers, immigrant and refugee children. Her focus is on pluralistic models that honour the role of culture in determining optimal caregiving. She has written extensively on the topics of human development and the situation of Latin American children and their families in Canada.

\section{Veronica Pacini-Ketchabaw}

Ontario Institute for Studies in Education

University of Toronto

Veronica Pacini-Ketchabaw is a native of Argentina. She is currently a doctoral candidate at the Ontario Institute for the Studies in Education of the University of Toronto and an instructor at the School of Early Childhood Education at Ryerson Polytechnic University in Canada. She is involved in a national study of multi-age approaches to early childhood education in contexts of cultural and linguistic diversity. she has worked with the Latin American community in Toronto in a variety of research projects. She is interested in how social relations of knowledge relate to educational issues confronting culturally and linguistically diverse populations.

\section{Marlinda Freire}

Hospital for Sick Children, University of Toronto

Marlinda Freire, M.D., F.R.C.P.(C) Chief Psychiatrist, Toronto District School Board. Assistant Professor, Faculty of Medicine, Department of Psychiatry, University of Toronto. Staff Psychiatrist, Hospital for Sick Children. Research Associate. Joint Centre of Excellence For Research on Immigration and Settlement, Federal Government of Canada. Research Associate. Centre for Refugee Studies, York University. Associate Fellow on Latin America and the Caribbean, York University. 